\title{
Fibrocyte and Keratinocyte-Like Cells and Their Role in Burn Wound Healing
}

TA Curran and A Ghahary*

Department or Surgery, Division of Plastic Surgery, University of British Columbia, Canada

\begin{abstract}
Burn injuries affect millions of people every year and dermal fibrosis is a common complication for the victims. This disfigurement has functional and cosmetic consequences and many research groups have made it the pivot of their work to understand the mechanisms that underlie its development. Although a significant amount of progress has been made in wound healing processes, the complexity of events involved makes it very difficult to come up with a single strategy to prevent this devastating fibrotic condition. Inflammation is considered one pre-disposing factor although this phase is a necessary aspect of the wound healing process. Inflammation, driven by infiltrated immune cells begins minutes after the burn injury and is the prevalent phase of wound healing in the early stages. Accompanying the inflammatory infiltrate there is evidence that sub-populations of bone marrow derived cells are also present. These populations include fibrocytes and keratinocyte-like cells, derivatives of CD14+ monocytes a component of the peripheral blood mononuclear cell infiltrate. There is evidence that these cells contribute to regeneration and repair of the wound site but interestingly there are also reports that these cells can have adverse effects and may contribute to the development of dermal fibrosis. We present here a review of the transdifferentiation and origin of these cells from bone marrow stem cells, the environments that direct this transdifferentiation and evidence to support their role in fibrosis as well as potential avenues for therapeutics to control their fibrotic effects.
\end{abstract}

Keywords: Fibrocytes; KLC; CD14+ Monocytes; Dermal fibrosis; Burn injuries

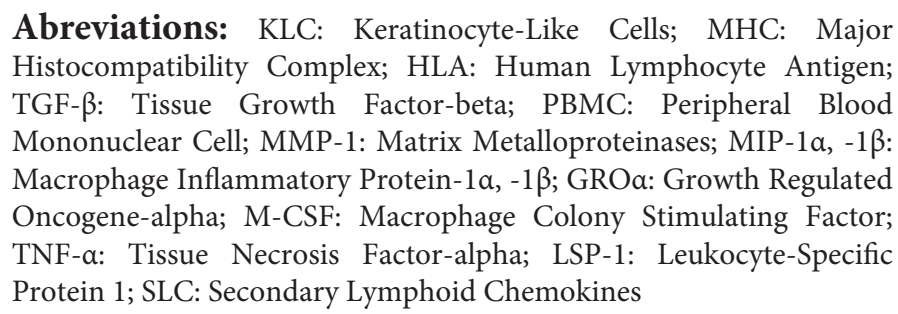

\section{Introduction}

Burn injuries cause disability and disfigurement in millions of people every year and result in more than 300,000 deaths worldwide [1]. These patients constitute an enormous proportion of the reconstructive work-load that is managed by plastic surgeons though-out the globe. The consequences of a burn injury can be lifelong for the patient with the potential for multiple surgeries, initially to cover the burn site and then to release contractures or for cosmesis, physiotherapy and a cocktails of medications. Aside from the physical effects, the individuals that survive often experience psychological sequelae as a result of disability and disfigurement [2]. From a molecular prospective the destruction of the skin and deeper tissues as a result of the burn stimulate the release of chemotactic factors from the remaining resident cells that initiate migration of various inflammatory cells into the wound. These migrated cells release factors that induce regeneration of the tissue by replenishing the cells populations and synthesizing extracellular components [3]. One possible sequelae of a burn injury is the risk of development of a hypertrophic or keloid scar $[4,5]$. The mechanisms that drive this are unclear although an association with delayed reepithelization and excessive matrix synthesis has been established $[4,6]$. Recent investigations have indicated that newly isolated bone marrow derived cells like fibrocytes and keratinocyte-like-cells (KLC) may be members of this inflammatory cell infiltrate during wound healing $[7,8]$. Recent evidence has suggested that these cells may play a role in the reepithelization process and importantly may also contribute to the development of dermal fibrosis in disordered wound healing $[8,9]$. In this review we present a synopsis of the transdifferention origin of these newly isolated cell populations, the evidence that supports their role in wound healing after burn injuries and their possible detrimental involvement in hyper proliferative disorders.

\section{Transdifferentation of Fibrocytes from Infiltrated Immune Cells}

Transdifferentiation is the term used to describe when cells change lineage commitment. This is predominantly a feature of stem cells and importantly the terminal differentiation phenotype can change depending on the environment of the stem cell. Fibrocytes and KLC transdifferentiate from adherent CD14+ monocytes, a peripheral blood mononuclear cell sub-population $[8,10]$. CD14+ monocytes also have the capacity to transdifferentiate into myofibroblasts [11], osteoblasts, skeletal myoblasts, chrondrocytes, adipocytes [12] and cardiomyocytes [13]. Fibrocytes were first described in 1994 by Bucala and colleagues who noted the presence of a large number of adherent, fibroblast-like spindle-shaped cells that infiltrated wound chambers, a model for tissue reparative responses in vivo, shortly after implantation. In addition to staining positive for collagen I, collagen III and vimentin these cells were also noted to express CD34, a surface receptor for hematopoietic stem cells [10]. Transdifferentiation into fibrocytes in vitro can be induced by culturing CD14+ monocytes in fibroblast culture medium [7]. In vivo fibrocytes are found in sites of tissue injury by day 4 post injury in tandem with an influx of inflammatory cells. This migration is believed to be mediated by fibrocyte expression of the chemokine receptor CCR7 which allows interaction with lymphoid chemokines,

*Corresponding author: Aziz Ghahary, PhD, Director, BCPFF Burn and Wound Healing Research Lab, 4th Floor, The Blusson Spinal Cord Centre, 818 West 10th Ave, Vancouver, BC, Canada, V5Z 1MQ, Tel: 604-675-8864, E-mail: aghahary@interchange.ubc.ca

Received December 14, 2011; Accepted January 10, 2012; Published January 19, 2012

Citation: Curran TA, Ghahary A (2012) Fibrocyte and Keratinocyte-Like Cells and Their Role in Burn Wound Healing. Surgery 2:106. doi:10.4172/2161-1076.1000106

Copyright: @ 2012 Curran TA, et al. This is an open-access article distributed under the terms of the Creative Commons Attribution License, which permits unrestricted use, distribution, and reproduction in any medium, provided the original author and source are credited. 
and fibrocytes will continue to accumulate in the wound until day 9-10 $[14,15]$.

\section{Transdifferentiation of Keratinocyte-Like Cells From Infiltrated Immune Cells}

Incidentally transdifferentiation of CD14+ cells to a KLC population can be encouraged in the presence of keratinocyte specific medium or by stimulation with bone morphogenetic protein-4 stimulation (BMP-4) in a 7day culture [3,8]. KLC are morphologically similar to keratinocytes, albeit with larger elliptical nuclei, and express

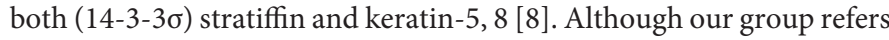
to these keratinocyte precursor cells as KLC, other groups have chosen to call them bone-marrow derived keratinocyte precursor cells, in addition to using different methods to encourage transdifferentiation of stem cell subsets; we have used transdifferentation from a CD14+ monocyte population but it is possible to derive these cells directly from CD34+ expressing bone marrow cells without prior selection of CD14+ cells $[3,16,17]$. Additional detection markers for the presence of KLC include positive staining for keratin-14 with negative expression of CD45. Chemotaxis of peripheral blood mononuclear cells, including the $\mathrm{CD} 14+$ monocytes to the wound site is regulated by the secretion of chemokines, CXCL5 and cytokines, $\beta$ fibroblast growth factor ( $\beta$ FGF) by fibroblasts located at the wound site [18]. Resident keratinocytes in the wound also appear to play a role by secretion of secondary lymphoid tissue chemokine (SLC/CCL21) which binds to the chemokine receptor CCR7 expressed on the mesenchymal stem cells [3].

\section{Fibrocytes and Wound Healing}

Fibrocytes make up $0.5 \%$ of peripheral blood leukocytes, but constitute $10 \%$ of cells infiltrating subcutaneously implanted chambers in mice which suggest that these cells have an important contribution in wound repair and regeneration [10]. They can behave as antigen presenting cells through tenure of MHC-class II and express the costimulatory molecules CD80, CD86 and adhesion molecules CD11a, CD54 and CD58 [19]. This contrasts with their mature fibroblast phenotype which requires pre-stimulation with IFN- $\gamma$ to express measurable quantities of HLA-DR [20]. Transdifferentiation of the CD14+ monocytes to fibrocytes appears to be dependent on the presence of CD14- cells but can also be induced through addition of CD14- conditioned medium alone. TGF- $\beta 1$ anti-serum blocks this effect suggesting it is the defining stimulator cytokine [21]. This correlates with the increased serum concentrations of TGF- $\beta 1$ in patients with large burn injuries and their high percentage of fibrocytes in PBMC cultures compared to controls [21]. Fibrocyte plasticity is evidenced by their ability to change fibrotic commitment. Fibrocytes express high levels of mRNA for the fibrogenic growth factors- platelet derived growth factor A (PDGF A) and TGF- $\beta 1$, hematopoietic growth factor, M-CSF, and chemokines MIP-1 $\alpha$ and MIP-2 [22]. Low levels of pro-inflammatory cytokines IL- $1 \alpha$ and TNF- $\alpha$ can also be detected. Our group has shown that fibrocytes derived from PBMCs can facilitate a switch in the fibro-proliferative commitment of recruited cells through modulation of MMP-1 expression and proliferation in a TGF- $\beta$ deprived culture. This suggests that fibrocytes are not irreversibly committed to the fibrogenic process [23]. These findings raise the possibility that after appropriate stimulation at the wound milieu, fibrocytes could express different profiles to work synergistically with specialised cells in a variety of functions. To confirm this hypothesis, Medina and Ghahary [7] injected bone marrow derived stem cells and precursor cells into adipofascial groin flaps of rats some of which were treated with anti-TGF- $\beta$ type II receptor neutralising antibody to induce unresponsiveness to bone marrow cells. Flaps from sham and bone marrow cell groups were notably denser and heavier with more fibrotic adherence to surrounding tissues compared to the antiTGF- $\beta$ group [7]. Histologically there was significantly more collagen deposition in the flaps from sham and bone marrow cell treated rats, than the anti-TGF- $\beta$ group. Instead flaps from the anti-TGF- $\beta$ group displayed a collagen pattern similar to normal adipofascial tissue collected from contralateral rat groins. There is now some evidence suggesting that overpopulation of wound beds with fibrocytes can lead to over healing or fibrotic conditions including hypertrophic scarring and keloid formation $[19,24,25,10]$.

\section{Inflammation and Fibrocyte Driven Fibrosis}

Appreciating that fibrocytes migrate to the wound site along with inflammatory cells it is not surprising that inflammation has a regulatory effect on fibrocyte activity. For example, stimulation of fibrocytes in vitro with wound healing mediator IL- $1 \beta$ suppresses type I collagen synthesis [24] but induces secretion of macrophage inflammatory protein-1 $\alpha$ (MIP-1 $\alpha)$, macrophage inflammatory protein-1 $\beta$ (MIP-1 $\beta$ ), monocyte chemo attractant protein-1 (MCP1 ), IL- 8 and growth regulated oncogene- alpha (GROa), IL-6 and macrophage colony stimulating factor (M-CSF), tissue growth factor$\beta 1$ (TGF- $\beta 1$ ) and tissue necrosis factor- $\alpha$ (TNF- $\alpha$ ) which incites migration of more inflammatory cells to the wound bed [22]. Prolonged inflammation seen in patients with large burn injuries correlates with an up regulation in fibrocyte differentiation compared to controls; moreover differentiated fibrocytes are seen to persist for longer at the burn site. A greater percentage of collagen producing fibrocytes are found in cultures of PBMC from burn patients compared to controls [21]. The inflammatory environment can additionally induce changes in morphology and the gene expression profile in fibrocytes $[10,14]$. Leukocyte-specific protein 1 (LSP-1) is highly expressed in fibrocytes and in combination with type I procollagen, can be used as a marker of fibrocytes [26]. LSP-1 up regulation in fibrocytes is presumably related to the adoption of an adherent phenotype and distinguishes them from their leukocyte companions in the inflammatory milieu. Prolonged inflammation in a wound is a predisposing factor for fibrosis and fibrocytes are found in significant numbers in all postburn hypertrophic scar tissue samples compared to non-hypertrophic scar tissue [26] (Figure 1 [24]). Uncontrolled accumulation of fibrocytes may thus play a significant role in the development and perpetuation

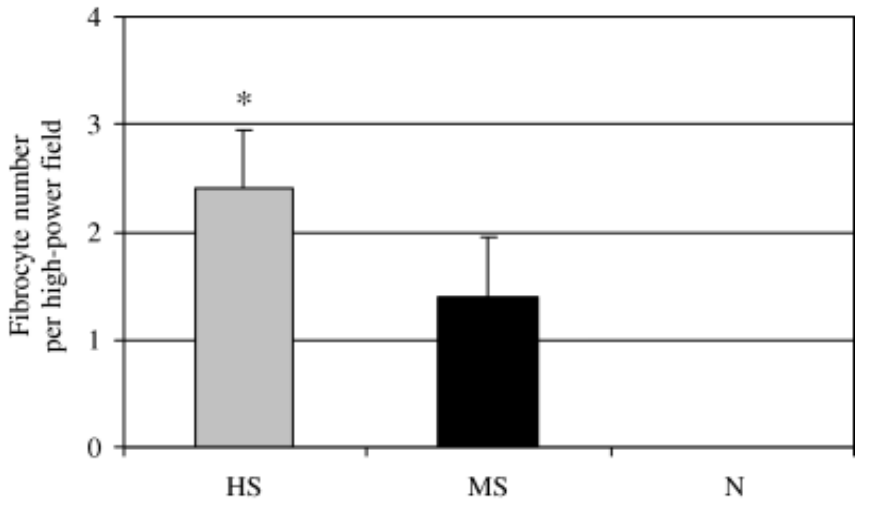

The number of dual-labelled fibrocytes per high-power field in the dermis was compared using one-way analysis of variance $\left({ }^{*} p<0.05\right)$. Courtesy Yang et al. [26]

Figure 1: Immunofluorescent staining for fibrocytes present in cryosections of hypertrophic scar (HS), Mature scar (MS) and normal skin (N) harvested from 10 burn patients. 
of hypertrophic scarring through differentiation into fibroblasts that express collagen and promotion of collagen release by local fibroblasts $[26,13]$.

\section{Keratinocyte-like Cells in Wound Healing}

Peripheral blood mononuclear cells (CD34+ cells) migrate to the wound site and are stimulated to transdifferentiate into keratinocytelike cells via interaction with cutaneous $\mathrm{T}$-cell attracting chemokine (CTACK/CCCL27) ligand which is up regulated in wounded tissue and the receptor CCR10 [16]. Another mechanism of bone marrow derived keratinocyte migration (CD34- cells) to the site of injury is through interaction with secondary lymphoid chemokine (SLC/CCL21) [3]. Our group has shown that CD14+ monocytes can also differentiate into KLC in keratinocyte specific medium $[3,8]$. We found that KLC conditioned cell medium alone can influence matrix synthesis in dermal fibroblasts, through the release of exosomes, and induce greater synthesis of matrix metalloproteinase-1 (MMP-1) release compared to fibroblasts cultured in keratinocyte conditioned medium [9] (Figure 2). Depletion of 14-3-3 proteins in the KLC conditioned medium inhibits this effect which suggests a pivotal role for 14-3-3 in the anti-fibrogenic response in KLC- dermal fibroblast co-culture [9]. Additionally keratinocytes reside in the hair follicle infundibulum and can assist in wound reepithelization, migrating into the wound between days 2 and 4 post wounding [27]. This evidence is supported by the fact that deep burn wounds, where the hair follicles have been destroyed are slower to heal [28].

\section{Application in Plastic Surgery}

Recently bone marrow, peripheral blood and umbilical cord blood have all been used as topical therapies to modulate the healing response in dermal wounds and change the nature of chronic wounds to acute wounds [29-31]. It is possible that fibrocytes and KLC, which are

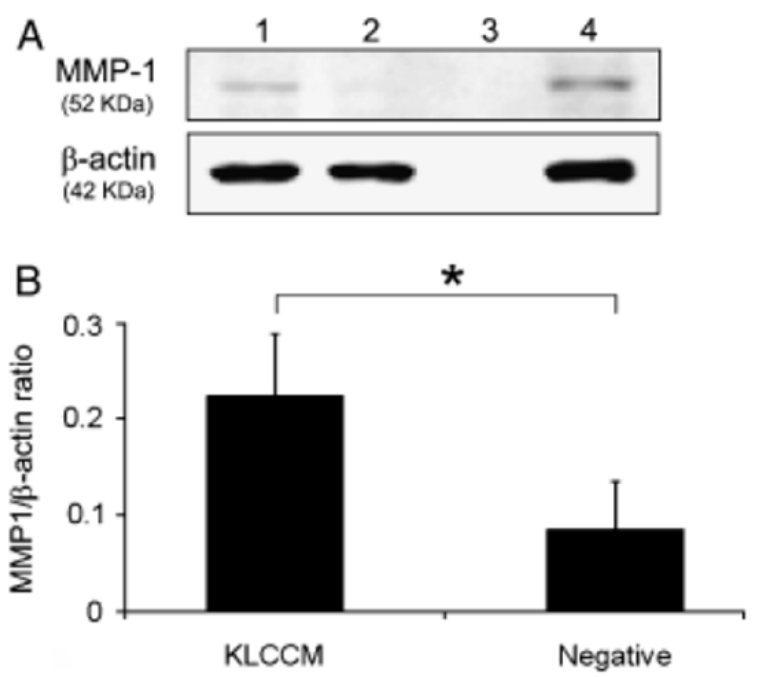

(A) Western blot of MMP-1 expression in dermal fibroblasts after 24 hours in either

$1=\mathrm{KLC}$ conditioned medium from day 28 of cell transdifferentiation $2=$ Dulbecco's modified Eagle medium + $2 \%$ FBS(negative control) $3=$ empty

$4=$ dermal fibroblasts treated with recombinant stratifin (positive control)

(B) Densitometry analysis of MMP-1/ $\beta$-actin ratio. $(n=4)$

Figure 2: Matrix metalloproteinase-1 (MMP-1) expression in dermal fibroblasts treated with $\mathrm{KLC}$ conditioned medium. stem cells derivatives, are involved. Stimuli initiates chemotaxis of the PBMC to the site of wound injury and chemokines at the wound bed induces transdifferentiation into fibrocytes or KLC. The morphological and genetic changes seen in the transdifferentiated CD14+ cells are dependent on the environment which can be unpredictable, in particular when considering a burn wound which can differ in depth and surface area. New research of the several chemokine channels that appear to drive MSC differentiation into KLC or fibrocytes offers a potential avenue for therapeutics. Obviously increased understanding of the mechanisms though which transdifferentiation is achieved is needed, especially regarding control of overpopulation of these subsets which studies have shown, may result in sub-optimal healing and excessive matrix deposition leading to hyper proliferative disorders.

\section{References}

1. WHO. Violence Injury prevention News.

2. WHO. Viloence injury prevention publications.

3. Sasaki M, Abe R, Fujita Y, Ando S, Inokuma D, et al. (2008) Mesenchymal stem cells are recruited into wounded skin and contribute to wound repair by transdifferentiation into multiple skin cell type. J Immunol 180: 2581-2587.

4. Deitch EA, Wheelahan TM, Rose MP, Clothier J, Cotter J (1983) Hypertrophic burn scars: analysis of variables. J Trauma 23: 895-898.

5. Page RE, Barclay TL (1981) Correlation of scald depth and hypertrophic scar formation. Burns 7: 173-175.

6. Robles DT, Berg D (2007) Abnormal wound healing: keloids. Clin Dermato 25: 26-32.

7. Medina A, Ghahary A (2010) Fibrocytes can be reprogrammed to promote tissue remodeling capacity of dermal fibroblasts. Mol Cell Biochem 344: 11-21.

8. Medina A, Brown E, Carr N, Ghahary A (2009) Circulating monocytes have the capacity to be transdifferentiated into keratinocyte-like cells. Wound Repair Regen 17: 268-277.

9. Medina A Ghahary A (2010) Transdifferentiated circulating monocytes release exosomes containing 14-3-3 proteins with matrix metalloproteinase-1 stimulating effect for dermal fibroblasts. Wound Repair Regen 18: 245-253.

10. Bucala R, Spiegel LA, Chesney J, Hogan M, Cerami A (1994) Circulating fibrocytes define a new leukocyte subpopulation that mediates tissue repair. Mol Med 1: 71-81.

11. Jabs A, Moncada GA, Nichols CE, Waller EK, Wilcox JN (2005) Periphera blood mononuclear cells acquire myofibroblast characteristics in granulation tissue. J Vasc Res 42: 174-180

12. Kuwana M, Okazaki Y, Kodama H, Izumi K, Yasuoka H, et al. (2003) Human circulating CD14+ monocytes as a source of progenitors that exhibit mesenchymal cell differentiation. J Leukoc Biol 74: 833-845.

13. Kodama H, Inoue $T$, Watanabe $R$, Yasuoka H, Kawakami $Y$, et al. (2005) Cardiomyogenic potential of mesenchymal progenitors derived from human circulating CD14+ monocytes. Stem Cells Dev 14: 676-686.

14. Abe R, Donnelly SC, Peng T, Bucala R, Metz CN (2001) Peripheral blood fibrocytes: differentiation pathway and migration to wound sites. J Immuno 166: 7556-7562.

15. Kao HK, Chen B, Murphy GF, Li Q, Orgill DP, et al. (2011) Peripheral blood fibrocytes: enhancement of wound healing by cell proliferation, reepithelialization, contraction, and angiogenesis. Ann Surg 254: 1066-1074

16. Inokuma D, Abe R, Fujita Y, Sasaki M, Shibaki A, et al. (2006) CTACK/CCL27 accelerates skin regeneration via accumulation of bone marrow-derived keratinocytes. Stem Cells 24: 2810-2816.

17. Medina A, Kilani RT, Carr N, Brown E, Ghahary A (2007) Transdifferentiation of peripheral blood mononuclear cells into epithelial-like cells. Am J Pathol 171 1140-1152.

18. Nedeau AE, Bauer RJ, Gallagher K, Chen H, Liu ZJ, et al. (2008) A CXCL5 and bFGF-dependent effect of PDGF-B-activated fibroblasts in promoting trafficking and differentiation of bone marrow-derived mesenchymal stem cells. Exp Cell Res 314: 2176-2186.

19. Chesney J, Bacher M, Bender A, Bucala R (1997) The peripheral blood 
Citation: Curran TA, Ghahary A (2012) Fibrocyte and Keratinocyte-Like Cells and Their Role in Burn Wound Healing. Surgery 2:106. doi:10.4172/21611076.1000106

fibrocyte is a potent antigen-presenting cell capable of priming naive T cells in situ. Proc Natl Acad Sci USA 94: 6307-6312.

20. Geppert TD, Lipsky PE (1985) Antigen presentation by interferon-gammatreated endothelial cells and fibroblasts: differential ability to function as antigen-presenting cells despite comparable la expression. J Immunol 135 3750-3762.

21. Yang L, Scott PG, Giuffre J, Shankowsky HA, Ghahary A, et al. (2002) Peripheral blood fibrocytes from burn patients: identification and quantification of fibrocytes in adherent cells cultured from peripheral blood mononuclear cells. Lab Invest 82: 1183-1192.

22. Chesney J,Bucala R (2001) Peripheral blood fibrocytes. In: Pallsson BO, Koller MR, Masters J. Kluwer Academic Publishers, London.

23. Medina A, Ghahary A (2010) Fibrocytes can be reprogrammed to promote tissue remodeling capacity of dermal fibroblasts. Mol Cell Biochem 344: 11-21.

24. Chesney J, Metz C, Stavitsky AB, Bacher M, Bucala R (1998) Regulated production of type I collagen and inflammatory cytokines by peripheral blood fibrocytes. J Immunol 160: 419-425.

25. Grab DJ, Lanners H, Martin LN, Chesney J, Cai C, et al. (1999) Interaction of Borreliaburgdorferi with peripheral blood fibrocytes, antigen-presenting cells with the potential for connective tissue targeting. Mol Med 5: 46-54.

26. Yang L, Scott PG, Dodd C, Medina A, Jiao H, et al. (2005) Identification of fibrocytes in postburn hypertrophic scar. Wound Repair Regen 13: 398-404.

27. Langton AK, Herrick SE, Headon DJ (2008) An extended epidermal response heals cutaneous wounds in the absence of a hair follicle stem cell contribution. J Invest Dermatol 128: 1311-1318.

28. Bishop GH (1945) Regeneration after experimental removal of skin in man. Am J Anat 76: 153-181.

29. Fathke C, Wilson L, Hutter J, Kapoor V, Smith A, et al. (2004) Contribution of bone marrow-derived cells to skin: collagen deposition and wound repair. Stem Cells 22: 812-822.

30. Han SK, Yoon TH, Lee DG, Lee MA, Kim WK (2005) Potential of human bone marrow stromal cells to accelerate wound healing in vitro. Ann Plast Surg 55 414-419.

31. Burd A, Ahmed K, Lam S, Ayyappan T, Huang L (2007) Stem cell strategies in burns care. Burns 33: 282-291. 